\title{
Abdominal wall reconstruction with component separation at the time of incisional hernia among survivors of emergency laparotomy after traumatic injuries: a population-based analysis of complications and healthcare utilization
}

\author{
Arturo J. Rios-Diaz',2, Jessica R. Cunning ${ }^{1}$, Joseph A. Mellia', Jesse Y. Hsu ${ }^{3}$, Cutler Whitely ${ }^{1}$, Robyn B. \\ Broach $^{1}$, John P. Fischer ${ }^{1}$ \\ 'Division of Plastic Surgery, Department of Surgery, University of Pennsylvania Health System, Philadelphia, PA 19104, USA \\ 2Division of Trauma and Acute Care Surgery, Department of Surgery, Thomas Jefferson University, Philadelphia, PA 19104, USA. \\ ${ }^{3}$ Center for Clinical Epidemiology and Biostatistics (CCEB), University of Pennsylvania, Philadelphia, PA 19104, USA.
}

Correspondence to: Dr. John P. Fischer, Division of Plastic Surgery, Department of Surgery, University of Pennsylvania Health System, 51 North 39th Street, Wright Saunders Building, Philadelphia, PA 19104, USA.

E-mail: John.Fischer2@pennmedicine.upenn.edu

\begin{abstract}
How to cite this article: Rios-Diaz AJ, Cunning JR, Mellia JA, Hsu JY, Whitely C, Broach RB, Fischer JP. Abdominal wall reconstruction with component separation at the time of incisional hernia among survivors of emergency laparotomy after traumatic injuries: a population-based analysis of complications and healthcare utilization. Plast Aesthet Res 2021;8:7. http://dx.doi.org/10.20517/2347-9264.2020.128
\end{abstract}

Received: 2 Jun 2020 First Decision: 17 Aug 2020 Revised: 18 Oct 2020 Accepted: 24 Nov 2020 Published: 21 Jan 2021

Academic Editors: Sahil Kuldip Kapur, Raúl González-García Copy Editor: Cai-Hong Wang Production Editor: Jing Yu

\begin{abstract}
Aim: The utilization and outcomes of abdominal wall reconstruction (AWR) using advanced techniques such as component separation for incisional hernia $(\mathrm{IH})$ repair following laparotomy in trauma populations has not been described. The objective was to describe AWR with component separation (AWR-CS) utilization in this setting and to assess postoperative complications and readmissions.
\end{abstract}

Methods: We identified adult patients admitted for IH repair (IHR) with a history of and admission for traumatic injuries with concurrent laparotomy in six geographically diverse statewide inpatient databases (2006-2015). AWR-CS was defined by ICD-9 codes corresponding to myocutaneous flap. Risk-adjusted logistic regression and generalized linear models were used to compare postoperative complications, 30-day readmissions and cumulative costs associated with AWR-CS.

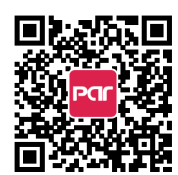


Results: Of 952 patients with a history of trauma laparotomy who were admitted electively for IHR, $6.8 \%$ underwent AWR-CS. Patients who underwent AWR-CS experienced increased complications [adjusted odds ratio 2.6 (95\%Cl: 1.48-4.57); $P<0.001$, cumulative costs (median $\$ 20,805$ vs. $\$ 15,529 ; P<0.001$ ) and longer length-ofstay (median days 6 vs. 5; $P=0.002$ ). These differences were driven by postoperative complication, which were independently associated with increased length of stay [predicted mean difference 6.53 days ( $95 \% \mathrm{Cl}: 4.66-8.41)$; $P<0.001]$, costs $[\$ 14,550$ (95\% Cl: \$ 9,258-19,841); $P<0.001]$ and 30-day cumulative costs $[\$ 20,176$ (95\% Cl: $\$ 12,621-27,731) ; P<0.001]$ within risk-adjusted analyses.

Conclusion: AWR-CS is part of the armamentarium needed to manage trauma laparotomy survivors who develop complex IH defects requiring surgical repair. It can result in increased complications that amplify postoperative healthcare utilization. Leverage of tools for the identification of high-risk patients, prehabilitation and enhanced surgical techniques is warranted to minimize postoperative complications in these patients.

Keywords: Flap, incisional hernia, trauma, laparotomy, complication, readmission, epidemiology, abdominal wall reconstruction

\section{INTRODUCTION}

According to data from the National Trauma Data Bank, over 6,000 patients undergo emergent laparotomy for traumatic injuries annually ${ }^{[1]}$, and $\mathrm{IH}$ can affect up to one-in-ten of these patients ${ }^{[2]}$. Incisional hernia (IH) often transforms into a chronic health state characterized by pain and discomfort that impairs functional status and quality of life ${ }^{[3,4]}$. In trauma patients, who may already face difficulties in recovery, this problem is a significant added burden. Complex IH defects representing large or fibrosed abdominal wall hernias often necessitate abdominal wall reconstruction (AWR) using flaps or component separation at the time of IH repair (IHR) to achieve myofascial closure and repair of the abdominal IH defect ${ }^{[5-7]}$.

Patients who survive life-saving laparotomies for traumatic injuries are prone to developing IH. Prior studies have shown that IH develops in $4 \%-10.5 \%$ of these patients ${ }^{[3,4]}$. In the emergent trauma setting, surgeons may be unable to perform a meticulous and careful abdominal closure and/or may need to delay primary closure due to hemodynamic instability or bowel edema encountered upon reoperation, all of which have the potential of increasing the risk for IH. Furthermore, in open abdomen cases, reapproximation of the abdominal wall may be challenging due to lateral migration of the rectus, decreased compliance of the oblique musculature, suboptimal skin availability, need for enterolysis and possible ostomy reversal. Therefore, surgeons may resort to the use of component separation techniques for AWR at the time of IHR. Although there are data on the utilization and outcomes of different AWR techniques at the initial trauma admission ${ }^{[8-11]}$, these utilization and outcomes data remain unknown for trauma laparotomy survivors who subsequently develop IH that necessitate surgical repair.

Given the little evidence available on AWR among trauma laparotomy patients, we leveraged longitudinal population data and sought to assess the postoperative outcomes and longitudinal healthcare utilization associated with the use of component separation for reconstruction of post-traumatic IH defects.

\section{METHODS}

\section{Study design and population}

This was a retrospective cohort study using statewide databases, the State Inpatient Databases (SID), corresponding to six states in diverse geographic regions of the United States: Florida (2006-2015), Iowa (2009-2015), Maryland (2013-2015), Nebraska (2006-2015), New York (2006-2015) and Wisconsin (20132015). These databases were used to identify adult patients ( $\geq 18$ years) admitted with traumatic injuries 


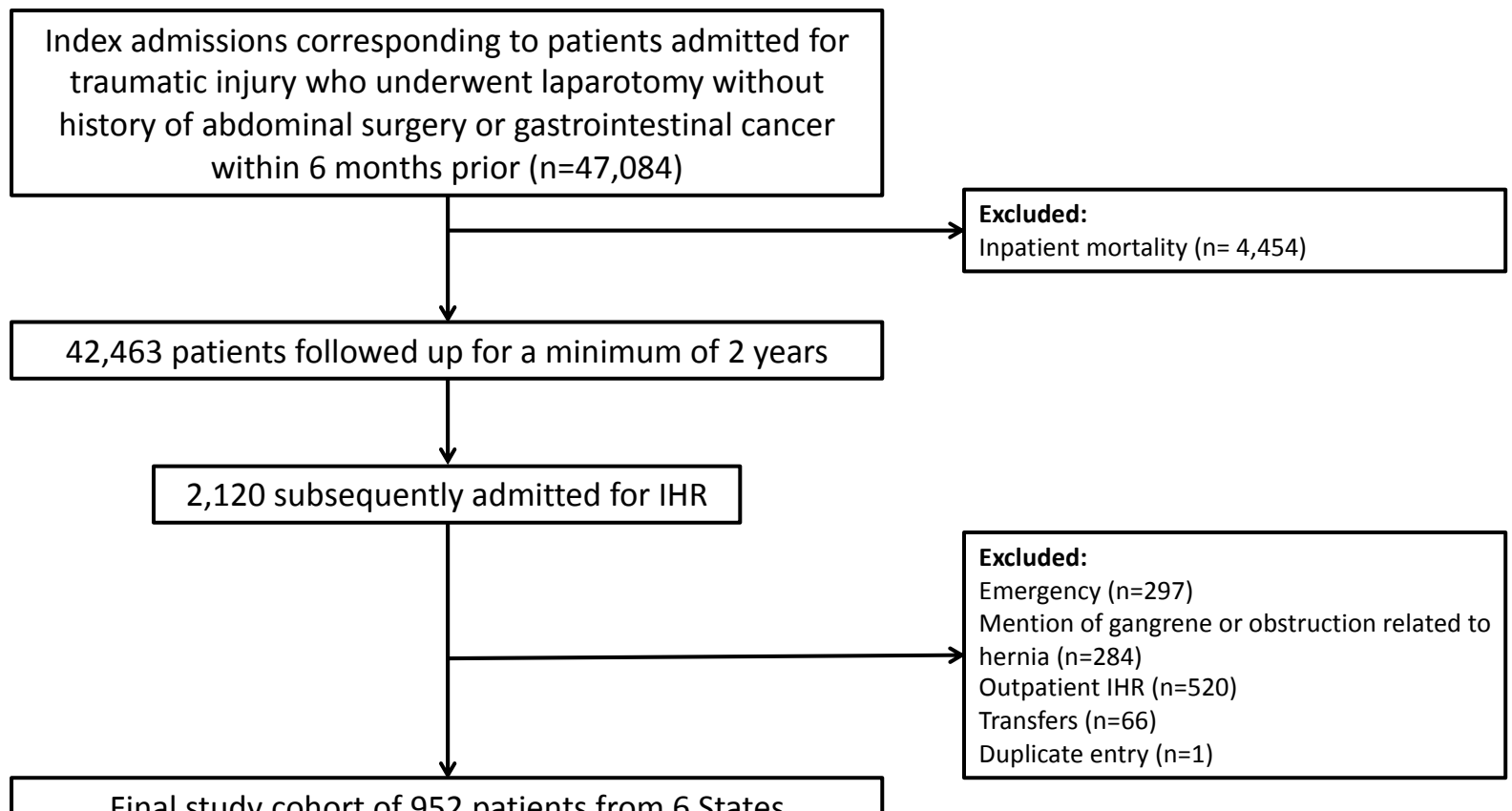

Final study cohort of 952 patients from 6 States

Figure 1. Flow diagram of inclusion and exclusion criteria. IHR: incisional hernia repair

who underwent laparotomy procedure(s) without a history of gastrointestinal cancer or prior abdominal procedure(s) within 6 months $(n=47,084)$. Those who were discharged alive $(n=42,463)$ were followed up for no less than 2 years until they presented for IHR in the inpatient setting (index encounter, Figure 1). Traumatic injuries were defined by the International Classification of Diseases, Ninth Revision, Clinical Modification (ICD-9-CM) diagnosis codes (800.xx-959.xx) with concurrent laparotomy procedure(s) as defined by ICD-9 procedure codes [Appendix 1]. Patients presenting from the Emergency Department (ED), those with a diagnosis of IH and incarceration or strangulation (ICD-9-CM 551.2X, 552.2X), and transfers between hospitals were excluded to eliminate potential treatment biases that may have occurred when managing IHR in patients that present under these circumstances. In addition, patients admitted in the last trimester of 2015 were excluded due to the transition from ICD-9-CM to ICD-10-CM. The primary exposure of interest was the use of AWR with component separation (AWR-CS) as defined by the use of myocutaneous flaps (ICD-9 procedure codes $86.7 \mathrm{X}$ ) adapted from previous methodology ${ }^{[12,13]}$.

\section{Data source}

We used multiple Agency for Healthcare Research and Quality Healthcare Cost and Utilization Project (HCUP) databases to conduct this study: SID, State Emergency Department Databases (SEDD) and State Ambulatory Surgery and Services Databases (SASD). The SID contains data on discharges for all patients admitted within the state ${ }^{[14]}$. The SEDD contains data on all ED discharges within the state that did not result in an admission, as well as observation stays ${ }^{[14]}$. The SASD contains data for ambulatory surgery and other outpatient encounters for hospital-owned facilities in participating states ${ }^{[15]}$. The SID was used to identify the trauma admission in which patients underwent laparotomy. The SID, SEDD and SASD were used to capture subsequent encounters until patients underwent IHR. Inpatient IHR was taken from SID encounters (index encounter) and outpatient IHR from the SASD, which were only used to quantify the proportion of outpatient IHR. Both the SID and SEDD were then used to capture subsequent encounters after the index admission, which were classified as readmissions. These datasets include clinical and nonclinical data for all patients regardless of age and insurance, including the uninsured, in addition to variables that permit conducting revisit analyses. Variables extracted include demographics, 
payer, comorbidities provided by the data, ICD-9-CM diagnosis and procedure codes, length of stay (LOS), and discharge disposition. In addition, ICD-9-CM codes permitted the identification of additional comorbidities and clinical outcomes not readily provided by the dataset (i.e., smoking status, postoperative complications such as pneumonia or surgical site infection).

\section{Demographic, clinical and hospital characteristics}

The demographic, clinical and hospital variables extracted included age, gender, race, insurance, zip code income quartile, state, Charlson Comorbidity Index, smoking status, obesity, HCUP-provided comorbidities (obesity, diabetes, hypertension, chronic lung disease, chronic heart failure, anemia, liver disease, renal failure, coagulopathy and weight loss), LOS, weekend admission, admission through the $\mathrm{ED}$, discharge disposition (home/routine; home with home health care; short-term hospital; skilled nursing facility (SNF), intermediate care facility (ICF) or another type of facility; against medical advice), laparoscopy use (53.62 and 53.63) and mesh use (53.6, 53.61, 53.63, 53.69).

\section{Outcome measures}

The primary outcome was a composite of the presence or absence of inpatient postoperative complications [non-surgical (cardiovascular, respiratory, genitourinary and thromboembolism), and surgical complications (surgical site infection, seroma, hematoma, delayed healing, wound disruption, fistula, accidental puncture, hemorrhage, blood transfusion, retained body, and reoperation)] as described elsewhere during the index encounter ${ }^{[16]}$. Secondary outcomes included prolonged LOS ( $>75$ th percentile) and non-routine discharge (other than home under self-care) for the index encounter, a composite of readmissions within 30 days of discharge (including ED visits, observation stays and subsequent inpatient admissions following surgery), costs during the index encounter and cumulative inpatient costs within 30 days.

\section{Estimation of costs}

The SID provides data on charges per inpatient encounter. These were converted to costs using hospitalspecific cost-to-charge ratios provided by HCUP. Cumulative cost was defined as the cost of the index admission in addition to the cost of any inpatient readmissions within 30 days. For patients with multiple readmissions, the aggregate readmission costs were obtained. These costs were normalized to U.S. dollars in 2019 using the Consumer Price Index. The annual costs were calculated to determine factors independently associated with increased costs.

\section{Statistical analysis}

Frequency, proportions, medians and interquartile ranges (IQR) were used to summarize demographic characteristics, clinical characteristics and the outcomes of interest. Chi-squared and Kruskal-Wallis tests were used for the comparison of categorical and continuous variables associated with the use of myocutaneous flaps during IHR. Risk-adjusted logistic regression models with robust standard errors clustering by hospital were used to ascertain adjusted odds ratios (OR) with $95 \% \mathrm{CI}$ for binary outcomes of interest (overall complications, surgical complications, non-surgical complications, non-routine discharge, prolonged length of stay and 30-day readmissions). Modified Park tests were used to determine the appropriate family distribution for each generalized linear model with $\log \operatorname{link}^{[17]}$, which was followed by post-estimation calculations of average marginal effects to ascertain the predicted mean differences in index LOS, index costs and 30-day cumulative costs with 95\%CI. Covariates in the models were identified a priori based on clinical judgement and known factors associated with complications during IHR. Statistical significance was set at $P<0.05$. All analyses were performed using StataMP Statistical Software, Release 15 (College Station, TX). The present study was approved by the University of Pennsylvania Health System Institutional Review Board. 


\section{RESULTS}

After inclusion and exclusion criteria were applied, there were 952 patients with a history of trauma laparotomy who were subsequently admitted electively for IHR; these patients comprised the analysis cohort. AWR-CS was used in $6.8 \%$ of these and mesh in $76.4 \%$. Overall, patients had a median age of 53 years (IQR 42-65), and the majority were male (64.4\%), white (70.2\%) and publicly insured (54.5\%). Patients who underwent IHR with AWR-CS reconstruction were more likely to be younger (median age 47.0 vs. 54.0; $P=0.044)$ and less likely to have undergone a laparoscopic IHR $(P=0.034)$. The rest of the assessed characteristics did not differ between groups (all $P>0.05$ ) and are shown in Table 1.

Table 2 shows the univariate comparison of adverse outcomes associated with the use of AWR-CS at the time of IHR. Patients in the AWR-CS group were more likely to experience overall $(57.4 \%$ vs. $40.1 \%$; $P=$ $0.008)$ and surgical complications $(45.9 \%$ vs. $26.2 \%$; $P<0.001)$. The breakdown of surgical complications is not shown in compliance with the data user agreement due to low counts. Among the surgical complications assessed, surgical site infection, delayed healing and wound disruption were higher in the AWR-CS group.

The overall readmission rate within 30 days was $23.7 \%$. Patients who had AWR-CS were more likely to experience 30 -day elective readmissions $(11.5 \%$ vs. $3.4 \% ; P=0.002)$ and reoperations during 30 -day readmission $(21.3 \%$ vs. 9.4\%; $P=0.003)$. Assessment of healthcare utilization outcomes in terms of LOS [median 6 days (IQR 4.0-8.0) vs. 5 (IQR 3.0-7.0); $P=0.002$ ], index admission costs [ $\$ 17,468.8$ (IQR $\$ 11,300.1-26,825.0)$ vs. $\$ 12,307.8$ (IQR $\$ 7,885.8-20,377.0) ; P<0.001]$ and cumulative costs [ $\$ 20,804.9$ (IQR \$ 16,145.1-34,941.0) vs. \$ 15,529.2 (IQR \$ 9,430.4-25,936.5); $P<0.001$ ] were increased for patients with a flap.

Within risk-adjusted analyses, patients with AWR-CS were $2.08(95 \%$ CI: $1.21-3.05 ; P=0.001)$ and 2.6 (95\%CI: $1.48-4.57 ; P<0.001)$ more likely to experience overall and surgical complications, respectively [Table 3]. Within univariate analyses, AWR-CS resulted in longer LOS, higher costs and higher cumulative costs. These differences were not statistically significant in risk-adjusted analyses after controlling for patient-level confounders $(P>0.05$; Table 4$)$. Within these models, the stronger predictors were complications, increasing the LOS by 6.53 days [predicted mean difference (95\%CI: 4.66-8.41); $P<0.001$ ], costs by $\$ 14,549.83$ (95\%CI: $\$ 9,258.82-19,840.84 ; P<0.001$ ) and cumulative costs by $\$ 20,175.85$ (95\%CI: $\$ 12,621.12-27,730.58 ; P<0.001)$. Based on the last estimate, the annualized cumulative cost associated with complications for the entire cohort was $\$ 790,893.32$.

\section{DISCUSSION}

IH is an under explored yet important long-term complication among survivors who undergo laparotomies for traumatic injuries. In these patients, successful IHR can be difficult due to a history of open abdomen and reoperations that often cause distorted abdominal wall anatomy (i.e., limited tissue elasticity, migration of the rectus abdominis muscle and decreased compliance of the oblique musculature). Given the complexity of IH after a trauma laparotomy, AWR with component separation may be required in some cases to achieve a durable abdominal closure while improving health-related quality of life ${ }^{[18]}$. This technique is often used in complex situations ${ }^{[19]}$ and requires a multidisciplinary approach that includes anesthesiologists, general surgeons and plastic surgeons ${ }^{[20]}$ to allow additional fascial approximation that would not be possible otherwise. Such an approach is also associated with lower IH recurrence rates ${ }^{[21]}$. In this population-based study using data from six states, we identified and described the clinical outcomes and healthcare utilization when flap reconstruction is performed for IHR in a sample of 952 patients that had undergone open abdominal surgery procedure(s) for traumatic injuries. We found that AWRCS was utilized in $6.8 \%$ of these cases and was associated with increased overall complications that result 
Table 1. Clinical-case mix and operative characteristics of patients with a history of trauma laparotomy undergoing incisional hernia repair, by use of abdominal wall reconstruction with component separation

\begin{tabular}{|c|c|c|c|}
\hline Characteristics & $\begin{array}{c}\text { No AWR-CS }(n=891) \\
n(\%)\end{array}$ & $\begin{array}{c}\text { AWR-CS }(n=61) \\
n(\%)\end{array}$ & $P$-value \\
\hline Age in years at admission, median (IQR) & $54.0(42.0,65.0)$ & $47.0(38.0,57.0)$ & $0.044^{\#}$ \\
\hline Indicator of sex & $320(35.9 \%)$ & $19(31.1 \%)$ & 0.57 \\
\hline Female & $396(37.5 \%)$ & $26(34.2 \%)$ & 0.45 \\
\hline \multicolumn{4}{|l|}{ Race } \\
\hline White & $625(70.1 \%)$ & $44(72.1 \%)$ & 0.36 \\
\hline Black & $90(10.1 \%)$ & * & \\
\hline Hispanic & $80(9.0 \%)$ & * & \\
\hline Other & $40(4.5 \%)$ & * & \\
\hline Not reported & $56(6.3 \%)$ & * & \\
\hline Insurance & & & 0.19 \\
\hline Medicare & $320(35.9 \%)$ & $17(27.9 \%)$ & \\
\hline Medicaid & $170(19.1 \%)$ & $12(19.7 \%)$ & \\
\hline Private & $303(34.0 \%)$ & $29(47.5 \%)$ & \\
\hline Self-pay & $20(2.2 \%)$ & * & \\
\hline No charge/Other & $78(8.8 \%)$ & * & \\
\hline \multicolumn{4}{|c|}{ Median household income state quartile for patient ZIP Code } \\
\hline 1st (lowest) & $217(24.7 \%)$ & $13(21.3 \%)$ & 0.35 \\
\hline 2nd & $230(26.2 \%)$ & $11(18.0 \%)$ & \\
\hline 3rd & $243(27.6 \%)$ & $21(34.4 \%)$ & \\
\hline 4th (highest) & $189(21.5 \%)$ & $16(26.2 \%)$ & \\
\hline Smoker & $263(29.5 \%)$ & $15(24.6 \%)$ & 0.41 \\
\hline Obese & $121(13.6 \%)$ & * & 0.052 \\
\hline Diabetes & $120(13.5 \%)$ & * & 0.78 \\
\hline Hypertension & $387(43.4 \%)$ & $24(39.3 \%)$ & 0.53 \\
\hline Chronic pulmonary disease & $157(17.6 \%)$ & $11(18.0 \%)$ & 0.93 \\
\hline Congestive heart failure & $30(3.4 \%)$ & * & 0.15 \\
\hline Deficiency anemias & $83(9.3 \%)$ & * & 0.07 \\
\hline Liver disease & $27(3.0 \%)$ & * & 0.53 \\
\hline Renal failure & $49(5.5 \%)$ & * & 0.73 \\
\hline Coagulopathy & $19(2.1 \%)$ & * & 0.80 \\
\hline Weight loss & $39(4.4 \%)$ & * & 0.30 \\
\hline Charlson Comorbidity Index & & & 0.48 \\
\hline$\leq 2$ & $843(94.6 \%)$ & $59(96.7 \%)$ & \\
\hline$>2$ & $48(5.4 \%)$ & * & \\
\hline Mesh use & $678(76.1 \%)$ & $49(80.3 \%)$ & 0.45 \\
\hline Laparoscopy use & $87(9.8 \%)$ & * & $0.034^{\#}$ \\
\hline \multicolumn{4}{|l|}{ Discharge disposition } \\
\hline Routine & $679(76.6 \%)$ & $41(67.2 \%)$ & 0.18 \\
\hline Transfer to short-term hospital & ${ }^{*}(0.1 \%)$ & $0(0.0 \%)$ & \\
\hline Transfer to SNF, ICF or other & $14(1.6 \%)$ & $0(0.0 \%)$ & \\
\hline Home health care & $192(21.7 \%)$ & $20(32.8 \%)$ & \\
\hline Weekend admission & $13(1.5 \%)$ & $\star$ & 0.91 \\
\hline
\end{tabular}

\#Statistically significant p-values less than 0.05. ${ }^{*}$ Cells with counts equal or greater than 10 are suppressed in compliance with HCUP data user agreement. IQR: interquartile ranges; SNF: skilled nursing facility; ICF: intermediate care facility; AWR-CS: abdominal wall reconstruction with component separation

in increased healthcare utilization in terms of LOS and costs. These complications were due to surgical wound-related complications for the most part, which is in line with prior data ${ }^{[18]}$.

AWR-CS at the time of IHR was independently associated with adverse postoperative outcomes. In this cohort, the surgical complication rate was $45.9 \%$, which rose to $57.4 \%$ when other non-surgical complications were considered. These results are within the complication range (12\%-67\%) reported after component separation in non-trauma populations ${ }^{[5,10,22-30]}$. Further examination revealed that the increase 
Table 2. Postoperative outcomes and healthcare utilization in patients with a history of trauma laparotomy undergoing incisional hernia repair, by use of abdominal wall reconstruction with component separation

\begin{tabular}{|c|c|c|c|}
\hline Outcome & $\begin{array}{c}\text { No AWR-CS }(n=891) \\
n(\%)\end{array}$ & $\begin{array}{c}\text { AWR-CS }(n=61) \\
n(\%)\end{array}$ & $P$-value \\
\hline Overall complications & $357(40.1 \%)$ & $35(57.4 \%)$ & $0.008^{\#}$ \\
\hline Non-surgical complications & $203(22.8 \%)$ & $17(27.9 \%)$ & 0.36 \\
\hline Surgical complications & $233(26.2 \%)$ & $28(45.9 \%)$ & $<0.001^{\#}$ \\
\hline Surgical site infection & $46(5.2 \%)$ & * & $0.009^{\#}$ \\
\hline Wound disruption & * & * & $<0.001^{\#}$ \\
\hline Reoperation & $46(5.2 \%)$ & $\star$ & 0.93 \\
\hline Non-routine discharge & $212(23.8 \%)$ & $20(32.8 \%)$ & 0.11 \\
\hline $\begin{array}{l}\text { Prolonged length of stay ( }>75 \text { th } \\
\text { percentile) }\end{array}$ & $166(18.6 \%)$ & $14(23.0 \%)$ & 0.40 \\
\hline 30-day readmission (Any) & $207(23.2 \%)$ & $19(31.1 \%)$ & 0.16 \\
\hline 30-day ED visit or observation stay & $86(9.7 \%)$ & $\star$ & 0.71 \\
\hline 30-day readmission (unplanned) & $177(19.9 \%)$ & $12(19.7 \%)$ & 0.97 \\
\hline 30-day readmission (elective) & $30(3.4 \%)$ & $\star$ & $0.002^{\#}$ \\
\hline $\begin{array}{l}\text { Reoperation during 30-day } \\
\text { readmission }\end{array}$ & $84(9.4 \%)$ & $13(21.3 \%)$ & $0.003^{\#}$ \\
\hline Index length of stay, median (IQR) & $5.0(3.0,7.0)$ & $6.0(4.0,8.0)$ & $0.002^{\#}$ \\
\hline Index costs (2019 USD), median (IQR) & $12307.8(7885.8,20337.0)$ & $17468.8(11300.1,26825.0)$ & $<0.001^{\#}$ \\
\hline $\begin{array}{l}\text { Cumulative costs ( } 2019 \text { USD), median } \\
\text { (IOR) }\end{array}$ & $15529.2(9430.4,25936.5)$ & $20804.9(16145.1,34941.0)$ & $<0.001^{\#}$ \\
\hline
\end{tabular}

\#Statistically significant p-values less than 0.05. ${ }^{*}$ Cells with counts equal or greater than 10 are suppressed in compliance with HCUP data user agreement. ED: Emergency Department; IQR: interquartile ranges; AWR-CS: abdominal wall reconstruction with component separation

Table 3. Logistic regression models showing the association of abdominal wall reconstruction with component separation with postoperative outcomes

\begin{tabular}{|c|c|c|c|c|}
\hline \multicolumn{5}{|l|}{ AWR-CS (Ref. No AWR-CS) } \\
\hline Outcome & OR & & & $P$-value \\
\hline Overall complications & 2.08 & 1.22 & 3.55 & $0.007^{\#}$ \\
\hline Systemic complications & 1.23 & 0.65 & 2.33 & 0.531 \\
\hline Surgery specific complications & 2.60 & 1.48 & 4.57 & $0.001^{\#}$ \\
\hline Reoperation & 0.84 & 0.24 & 2.97 & 0.784 \\
\hline Non-routine discharge & 1.69 & 0.97 & 2.96 & 0.066 \\
\hline Prolonged length of stay ( $>75$ th percentile) & 1.18 & 0.63 & 2.20 & 0.601 \\
\hline 30-day readmission (Any) & 1.47 & 0.80 & 2.69 & 0.213 \\
\hline
\end{tabular}

\#Statistically significant p-values less than 0.05. OR: odds ratios; IQR: interquartile ranges. Models controlled for age, gender, race, insurance, obesity, smoking, anemia, Charlson Comorbidity Index, mesh use and laparoscopy; AWR-CS: abdominal wall reconstruction with component separation

Table 4. Generalized linear models showing the association of abdominal wall reconstruction with component separation with differences in healthcare utilization

\begin{tabular}{|c|c|c|c|c|}
\hline \multicolumn{5}{|l|}{ AWR-CS (Ref. No AWR-CS) } \\
\hline Outcome & Predicted mean difference & & & $P$-value \\
\hline Length of stay, median (IQR) & -1.71 & -5.87 & 2.45 & 0.420 \\
\hline Costs (2019 USD), median (IQR) & $-\$ 2,102.08$ & $-\$ 9,187.68$ & $\$ 4,983.52$ & 0.561 \\
\hline Cumulative costs (2019 USD), median (IQR) & $-\$ 1,819.31$ & $-\$ 11,300.00$ & $\$ 7,710.05$ & 0.708 \\
\hline
\end{tabular}

OR: odds ratios; IQR: interquartile ranges. Models controlled for age, gender, race, insurance, obesity, smoking, anemia, Charlson Comorbidity Index, mesh use and laparoscopy; AWR-CS: abdominal wall reconstruction with component separation

in surgical complications was due to surgical site infections, delayed healing and wound disruption (data not shown due to data user agreement restrictions), which is in agreement with previous studies showing that wound complications are prevalent in these patients ${ }^{[2,26]}$. These high rates are likely due to the space created during the extensive and lengthy dissection and mobilization of the abdominal skin required by 
the component separation technique, which can affect perfusion of the flaps and leave a dissection plane that may be prone to seromas or hematomas and, thus, increase infections ${ }^{[2,31]}$. Furthermore, it is possible that this risk is compounded in the setting of trauma, where the abdominal wall anatomy may already be significantly altered from repeated disruption of its integrity, delayed closure, percutaneous drain(s) insertion and/or ostomy creation/reversal. Future studies should categorize the level of trauma to the abdominal wall for the purpose of stratifying the risks associated with performing AWR-CS. Despite AWRCS involving maneuvers that further distort anatomy and thereby increase the propensity for complications, the alternative approach used when primary fascial closure cannot be achieved, bridged repair, tends to yield poorer outcomes as evidence in prior literature ${ }^{[32]}$. Therefore, surgeons should still consider AWR-CS when clinically indicated.

It is important to highlight that survivors of trauma laparotomy represent a heterogenous group with varying patient-specific risk factors for AWR-CS and adverse outcomes. No traumatic injury is the same, and subsequent surgical management of these patients (the need for delayed abdominal closure, staged repair, etc.) may differ based on the nature of the initial injury (sharp vs. blunt, associated intrabdominal damage, etc.) and immediate outcomes after the initial interventions, such as early postoperative complications and need for multiple reoperations. In addition, since there is a temporal variation in the development of IH following trauma laparotomy, patients inevitably present at different stages of wound healing, adding another layer of complexity to the patient's profile. Therefore, each IH originating after trauma laparotomy may be "complex" due to a unique combination of factors and it is not surprising that AWR-CS at the time of IHR was associated with adverse outcomes. Slater et al ${ }^{[33]}$ provided a framework for understanding these patient-specific factors that contribute to the inherent complexity. Although there are many factors, some particularly relevant to trauma patients include emergency operation with bowel resection, open (burst) abdomen, and significantly distorted anatomy from trauma and/or multiple reoperations during the initial trauma management. The open abdomen, in particular, is one of the few stand-alone characteristics that surgeons agree make a complex hernia most severe ${ }^{[33]}$. Since this management technique is commonly used in trauma surgery, it is possible that trauma laparotomy survivors are more likely to have complex hernias of greater severity, thereby increasing their risk for adverse events after AWR-CS as compared to non-trauma complex hernia patients.

The findings of the present study have implications that involve various stakeholders. The increase in complications after IHR with AWR-CS in survivors of trauma laparotomy underscores the complexity of these cases undertaken often by experienced general and plastic and reconstructive surgeons, and highlights the need for additional research in this area. Surgeons should not be discouraged from using AWR-CS techniques at the time of IHR when clinically indicated but rather, focus their efforts in minimizing postoperative complications. This may be achieved by leveraging risk-prediction tools, as developed previously ${ }^{[34]}$, that provide actionable information that can be used to maximize health in preparation for surgery and therefore, minimize the risk for postoperative complications. Other strategies for the optimization of postoperative outcomes may include the enhancement of surgical technique through novel approaches such as component separation with chemodenervation ${ }^{[35]}$, or minimally invasive component separation with inlay bioprosthetic mesh $^{[36]}$. Furthermore, this study illustrates the magnitude of the increased risk associated with AWR-CS at the time of IHR, which can aid clinicians with counseling patients, setting realistic expectations, and optimizing shared decision-making. These results may also be used as future benchmarks for programs aiming to improve the quality of care provided, such as pay-forperformance and value-based purchasing ${ }^{[37]}$. Lastly, from the perspectives of the payer and public health, this study underscores the burden of IH after trauma laparotomy and the healthcare utilization burden in the following years when patients return for IHR (with and without AWR-CS).

There are several limitations to this study including its retrospective nature and the use of administrative data. We relied on billing codes, which render this study subject to misclassification and selection bias. 
However, we used an approach adapted from previously described methods for the identification of patients undergoing AWR-CS at the time of IHR and attempted to minimize selection bias by excluding patients admitted through the ED or for emergent indications of IHR (incarceration or strangulation) ${ }^{[12]}$. In addition, we were unable to control for more granular clinical data such as baseline functional status, size of the hernia defect, reconstructive details (i.e., anterior $v$ s. posterior component separation, open vs. minimally invasive component separation), wound classification and laboratory values as they are not available within the administrative databases used in this study. Furthermore, although our approach comprehensively captures post-discharge healthcare utilization through ED visits, observation stays and inpatient admissions after the index procedure, these data do not include clinic visits, which may represent a significant proportion of healthcare utilization after discharge. The healthcare cost estimates were limited to the population who were readmitted and met inpatient criteria given that the SEDD does not allow conversion of charges to costs. The purpose of this study was not to describe the incidence of IH among trauma laparotomy survivors. Therefore, the proportion of patients we identified upon elective admission is not a reflection of the incidence of $\mathrm{IH}$. This is due to the fact that a number of patients from the initial trauma cohort did not have more than two years of follow-up. Lastly, even though statewide data from diverse geographical regions were used, it is unclear whether the trends observed are generalizable to the entire population in the U.S. Nonetheless, this is the largest study to date examining AWR practice patterns and outcomes among trauma survivors who had undergone laparotomy due to their injuries.

The use of AWR-CS is part of the armamentarium for trauma laparotomy survivors who develop IH and require surgical repair. This approach is associated with increased complications that can result in increased healthcare costs and utilization. Surgeons should counsel patients accordingly and strive to maximize health preoperatively to reduce the risk of postoperative complications.

\section{DECLARATIONS}

\section{Acknowledgments}

Rios-Diaz AJ and Cunning JR would like to acknowledge support from the Center for Human Appearance at the University of Pennsylvania.

\section{Authors' contributions}

Concept and design: Rios-Diaz AJ, Broach RB, Fischer JP

Data acquisition and analysis: Rios-Diaz AJ, Hsu JY, Broach RB, Fischer JP

Data analysis: Rios-Diaz AJ, Hsu JY

Data interpretation, critical revisions: Rios-Diaz AJ, Cunning JR, Mellia JA, Hsu JY, Whitely C, Broach RB, Fischer JP

Manuscript drafting: Rios-Diaz AJ, Cunning JR, Mellia JA, Whitely C, Broach RB, Fischer JP

\section{Availability of data and materials}

Not appliable.

\section{Financial support and sponsorship}

Fischer JP has received consulting fees for educational speaking from Baxter, Becton Dickinson, Integra, and Gore.

\section{Conflicts of interest}

Fischer JP has received consulting fees for educational speaking from Baxter, Becton Dickinson, Integra, and Gore. The remaining authors do not have any conflicts of interest. 


\section{Ethical approval and consent to participate}

The present study was exempted from full review as per by the University of Pennsylvania Health System Institutional Review Board as the data used in the study is de-identified.

\section{Consent for publication}

Non-applicable.

\section{Copyright}

(c) The Author(s) 2021.

\section{REFERENCES}

1. Benson C, Weinberg J, Narsule CK, Brahmbhatt TS. A comparison of alcohol positive and alcohol negative trauma patients requiring an emergency laparotomy. Am J Emerg Med 2018;36:1139-44.

2. Li T, Robertson-More C, Maclean AR, et al. Bowel obstructions and incisional hernias following trauma laparotomy and the nonoperative therapy of solid organ injuries: a retrospective population-based analysis. J Trauma Acute Care Surg 2015;79:386-92.

3. van Ramshorst GH, Eker HH, Hop WC, Jeekel J, Lange JF. Impact of incisional hernia on health-related quality of life and body image: a prospective cohort study. Am J Surg 2012;204:144-50.

4. Snyder CW, Graham LA, Vick CC, Gray SH, Finan KR, Hawn MT. Patient satisfaction, chronic pain, and quality of life after elective incisional hernia repair: effects of recurrence and repair technique. Hernia 2011;15:123-9.

5. Ramirez OM, Ruas E, Dellon AL. "Components separation" method for closure of abdominal-wall defects: an anatomic and clinical study. Plast Reconstr Surg 1990;86:519-26.

6. Novitsky YW, Elliott HL, Orenstein SB, Rosen MJ. Transversus abdominis muscle release: a novel approach to posterior component separation during complex abdominal wall reconstruction. Am J Surg 2012;204:709-16.

7. Pauli EM, Rosen MJ. Open ventral hernia repair with component separation. Surg Clin North Am 2013;93:1111-33.

8. Poulakidas S, Kowal-Vern A. Component separation technique for abdominal wall reconstruction in burn patients with decompressive laparotomies. J Trauma 2009;67:1435-8.

9. Hultman CS, Pratt B, Cairns BA, et al. Multidisciplinary approach to abdominal wall reconstruction after decompressive laparotomy for abdominal compartment syndrome. Ann Plast Surg 2005;54:269-75; discussion 275.

10. Cohen M, Morales R, Fildes J, Barrett J. Staged reconstruction after gunshot wounds to the abdomen. Plast Reconstr Surg 2001;108:83-92.

11. Howdieshell TR, Proctor CD, Sternberg E, Cué JI, Mondy JS, Hawkins ML. Temporary abdominal closure followed by definitive abdominal wall reconstruction of the open abdomen. Am J Surg 2004;188:301-6.

12. Clarke TM, Goldberg RF, Lloyd JM, Rosales-Velderrain A, Bowers SP. The Increasing Utilization of Component Separation Technique During Ventral Hernia Repair: Association of Patient, Payor, and Community Demographics. Available from: https://www.sages.org/ meetings/annual-meeting/abstracts-archive/the-increasing-utilization-of-component-separation-technique-during-ventral-hernia-repairassociation-of-patient-payor-and-community-demographics/. [Last accessed on 8 Dec 2020]

13. Ferguson DH, Smith CG, Olufajo OA, Zeineddin A, Williams M. Risk factors associated with adverse outcomes after ventral hernia repair with component separation. J Surg Res 2020;258:299-306.

14. Agency for Healthcare Research and Quality. Healthcare Cost and Utilization Project (HCUP). Available from: https://www.hcup-us.ahrq. gov/db/state/siddbdocumentation.jsp. [Last accessed on 20 May 2020]

15. Healthcare Cost and Utilization Project (HCUP). Overview of the State Ambulatory Surgery and Services Databases (SASD). Available from: https://www.hcup-us.ahrq.gov/sasdoverview.jsp. [Last accessed on 8 Dec 2020]

16. Rios-Diaz AJ, Lam J, Zogg CK. The need for postdischarge, patient-centered data in trauma. JAMA Surg 2016;151:1101-2.

17. Norton EC, Dowd BE. Log odds and the interpretation of logit models. Health Serv Res 2018;53:859-78.

18. Maloney SR, Schlosser KA, Prasad T, et al. The impact of component separation technique versus no component separation technique on complications and quality of life in the repair of large ventral hernias. Surg Endosc 2020;34:981-7.

19. Zolper EG, Black CK, Devulapalli C, et al. Long term outcomes of abdominal wall reconstruction using open component separation and biologic mesh in the liver, kidney, and small bowel transplant population. Hernia 2020;24:469-79.

20. Kingsnorth AN, Sivarajasingham N, Wong S, Butler M. Open mesh repair of incisional hernias with significant loss of domain. Ann $R$ Coll Surg Engl 2004;86:363-6.

21. Maloney SR, Schlosser KA, Prasad T, et al. Twelve years of component separation technique in abdominal wall reconstruction. Surgery 2019;166:435-44.

22. DiBello JN, Moore JH. Sliding myofascial flap of the rectus abdominus muscles for the closure of recurrent ventral hernias. Plast Reconstr Surg 1996;98:464-9.

23. Girotto JA, Ko MJ, Redett R, Muehlberger T, Talamini M, Chang B. Closure of chronic abdominal wall defects: a long-term evaluation of the components separation method. Ann Plast Surg 1999;42:385-94; discussion 394-5.

24. Jernigan TW, Fabian TC, Croce MA, et al. Staged management of giant abdominal wall defects: acute and long-term results. Ann Surg 2003;238:349-55; discussion 355-7. 
25. Kuzbari R, Worseg AP, Tairych G, et al. Sliding door technique for the repair of midline incisional hernias. Plast Reconstr Surg 1998;101:1235-42.

26. Lowe JB, Lowe JB, Baty JD, Garza JR. Risks associated with "components separation" for closure of complex abdominal wall defects. Plast Reconstr Surg 2003;111:1276-83; quiz 1284-5; discussion 1286-8.

27. Shestak KC, Edington HJ, Johnson RR. The separation of anatomic components technique for the reconstruction of massive midline abdominal wall defects: anatomy, surgical technique, applications, and limitations revisited. Plast Reconstr Surg 2000;105:731-8; quiz 739.

28. Sukkar SM, Dumanian GA, Szczerba SM, Tellez MG. Challenging abdominal wall defects. Am J Surg 2001;181:115-21.

29. de Vries Reilingh TS, van Goor H, Rosman C, et al. "Components separation technique" for the repair of large abdominal wall hernias. $J$ Am Coll Surg 2003;196:32-7.

30. Ennis LS, Young JS, Gampper TJ, Drake DB. The "open-book" variation of component separation for repair of massive midline abdominal wall hernia. Am Surg 2003;69:733-42; discussion 742-3.

31. de Vries Reilingh TS, van Goor H, Charbon JA, et al. Repair of giant midline abdominal wall hernias: "components separation technique" versus prosthetic repair: interim analysis of a randomized controlled trial. World J Surg 2007;31:756-63.

32. Holihan JL, Askenasy EP, Greenberg JA, et al, Ventral Hernia Outcome Collaboration Writing Group. Component separation vs. bridged repair for large ventral hernias: a multi-institutional risk-adjusted comparison, systematic review, and meta-analysis. Surg Infect (Larchmt) 2016; 17:17-26.

33. Slater NJ, Montgomery A, Berrevoet F, et al. Criteria for definition of a complex abdominal wall hernia. Hernia 2014;18:7-17.

34. Basta MN, Kozak GM, Broach RB, et al. Can we predict incisional hernia? development of a surgery-specific decision-support interface. Ann Surg 2019;270:544-53.

35. Weissler JM, Lanni MA, Tecce MG, Carney MJ, Shubinets V, Fischer JP. Chemical component separation: a systematic review and metaanalysis of botulinum toxin for management of ventral hernia. J Plast Surg Hand Surg 2017;51:366-74.

36. Butler CE, Campbell KT. Minimally invasive component separation with inlay bioprosthetic mesh (MICSIB) for complex abdominal wall reconstruction. Plast Reconstr Surg 2011;128:698-709.

37. Center for Medicare \& Medicaid Services. The Hospital Value-Based Purchasing (VBP) Program. Available from: https://www.cms.gov/ Medicare/Quality-Initiatives-Patient-Assessment-Instruments/Value-Based-Programs/HVBP/Hospital-Value-Based-Purchasing. [Last accessed on 8 Dec 2020] 